\title{
Superfamilies and families of parasitoids (Hymenoptera) collected in the forest of Brazil.
}

Carlos Henrique Marchiori ${ }^{1}$

1 Instituto Federal Goiano

Interest in biological controls has grown in various countries, as a response to the adverse effects of chemical pesticides on the environment and on biodiversity. Moreover, it has grown as a function of new international trends in agricultural production involving the utilization of alternative means that are less aggressive to the environment and which favor conservation and the sustainable use of biodiversity

The objective of this study was to verify the superfamilies and families of parasitoids (Hymenoptera) collected in area of forest in the south Goiás, Brazil using yellow bowl traps February to October 2002.

Sampling was weekly, with 10 yellow bowl traps placed at ground level and allocated at random to areas of native vegetation and pastures. A total of five yellow pan traps were placed in the pastures and five in forests. These traps were the spherical yellow plastic bowls approximately $30 \mathrm{~cm}$ in diameter and $12 \mathrm{~cm}$ height which was deposited a mixture of 2 liters of water, $2 \mathrm{ml}$ of detergent and $2 \mathrm{ml}$ of formaldehyde (Figure).

List of Superfamilies and families of parasitoids (Hymenoptera) (Table 1). 


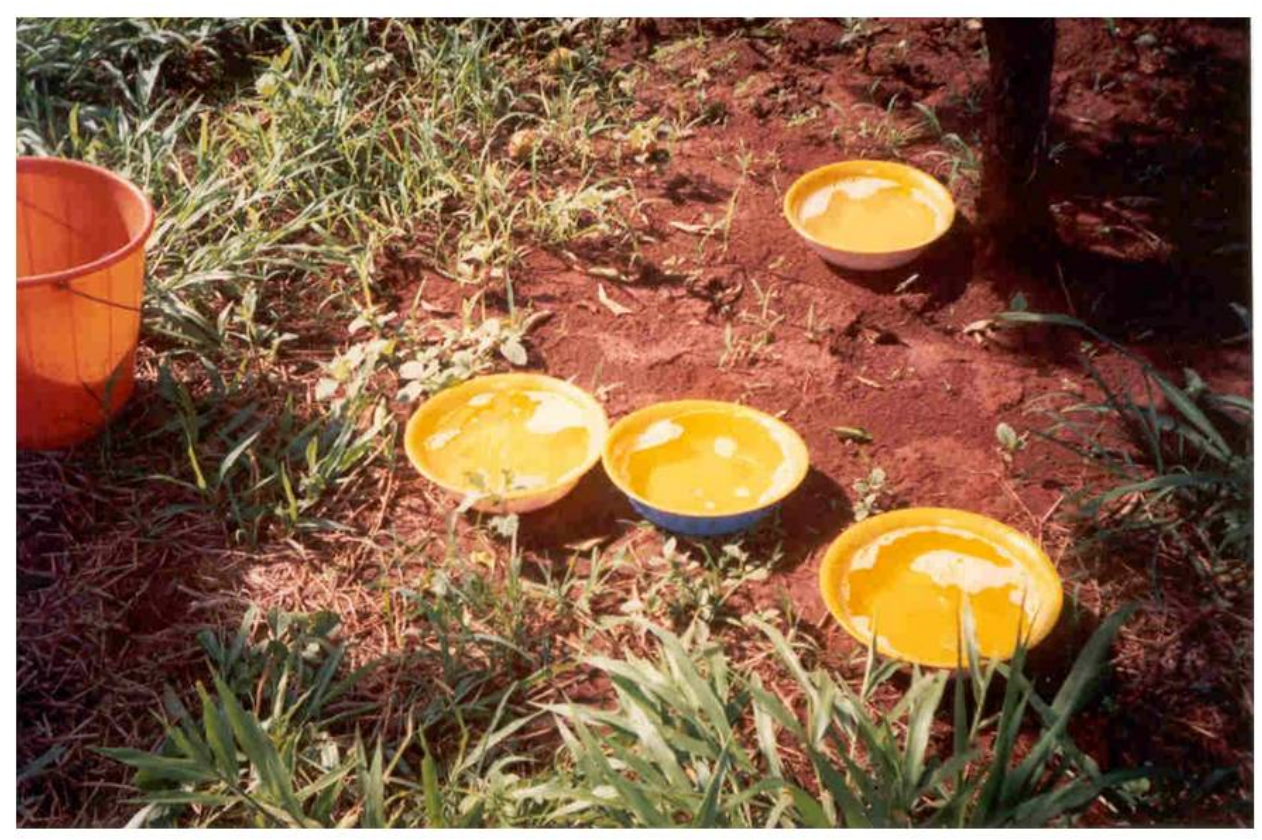

Figure - Yellow Bowl Traps

Figure

) .

Table - Superfamilies and families of parasitoids collected in the forest of Brazil in 2002.

\begin{tabular}{|c|c|c|c|c|c|c|c|c|c|}
\hline \multirow{2}{*}{$\begin{array}{c}\text { Taxonomic Group } \\
\text { Superfamilies/ } \\
\text { Families }\end{array}$} & \multicolumn{9}{|c|}{ Months } \\
\hline & Feb. & Mar. & Apr. & May & Jun. & Jul. & Aug. & Sep. & Oct. \\
\hline \multicolumn{10}{|l|}{ Bethylidoidea: } \\
\hline Bethylidae & 46 & 28 & 13 & 35 & 13 & 14 & 25 & 31 & 16 \\
\hline Dryinidae & 1 & 1 & 0 & 2 & 0 & 0 & 0 & 1 & 1 \\
\hline \multicolumn{10}{|l|}{ Ceraphranoidea: } \\
\hline Ceraphronidae & 16 & 20 & 31 & 13 & 2 & 2 & 5 & 9 & 1 \\
\hline Megaspilidae & 1 & 1 & 0 & 2 & 1 & 0 & 12 & 2 & 0 \\
\hline \multicolumn{10}{|l|}{ Chalcidoidea: } \\
\hline Agoanidae & 1 & 0 & 0 & 1 & 0 & 0 & 0 & 0 & 0 \\
\hline Chalcididae & 1 & 1 & 7 & 5 & 0 & 3 & 3 & 15 & 2 \\
\hline Encyrtidae & 118 & 31 & 24 & 89 & 35 & 40 & 13 & 17 & 3 \\
\hline Eucharitidae & 0 & 4 & 1 & 3 & 1 & 2 & 3 & 1 & 0 \\
\hline Eulophidae & 16 & 8 & 16 & 34 & 5 & 8 & 7 & 10 & 15 \\
\hline Eupelmidae & 6 & 1 & 4 & 2 & 3 & 3 & 13 & 26 & 13 \\
\hline Eurytomidae & 1 & 0 & 0 & 0 & 2 & 3 & 2 & 0 & 1 \\
\hline Mymaridae & 1 & 1 & 0 & 2 & 1 & 0 & 11 & 2 & 0 \\
\hline Pteromalidae & 9 & 0 & 2 & 12 & 3 & 8 & 3 & 0 & 1 \\
\hline Torymidae & 20 & 4 & 3 & 13 & 3 & 6 & 4 & 0 & 0 \\
\hline Cynipoidea: & & & & & & & & & \\
\hline Figitidae & 12 & 15 & 12 & 3 & 2 & 0 & 0 & 3 & 4 \\
\hline Evanoidea: & & & & & & & & & \\
\hline Evaniidae & 3 & 0 & 2 & 2 & 0 & 0 & 3 & 7 & 3 \\
\hline \multicolumn{10}{|l|}{ Ichneumonoidea: } \\
\hline Braconidae & 113 & 25 & 33 & 18 & 9 & 6 & 9 & 12 & 4 \\
\hline Ichneumonidae & 118 & 67 & 87 & 42 & 8 & 4 & 33 & 61 & 21 \\
\hline \multicolumn{10}{|l|}{ Platyga stroidea: } \\
\hline Platygastridae & 4 & 0 & 2 & 8 & 4 & 3 & 12 & 1 & 1 \\
\hline Scelionidae & 14 & 2 & 4 & 13 & 5 & 9 & 1 & 0 & 1 \\
\hline \multicolumn{10}{|l|}{ Proctotrupoidea: } \\
\hline Diapriidae & 106 & 12 & 65 & 50 & 22 & 18 & 19 & 48 & 10 \\
\hline
\end{tabular}

Figure 